\title{
NANOPARTICLE TECHNOLOGY TO DELIVER EFFECTIVE ANTIMICROBIALS
}

\author{
${ }^{1}$ Sarah E. Maddocks, ${ }^{2}$ Michele E. Barbour and ${ }^{3}$ Andrew M. Collins \\ ${ }^{1}$ Department of Biomedical Science, Cardiff School of Health Sciences, Cardiff Metropolitan University, UK \\ ${ }^{2}$ Oral Nanoscience, School of Oral and Dental Sciences, University of Bristol, UK \\ ${ }^{3}$ Bristol Centre for Functional Nanomaterials, University of Bristol, UK
}

Bacterial resistance to antimicrobials emerged only a few years after the commercial availability of antibiotics. Numerous factors have enabled the escalation of antimicrobial resistance to occur unabated, meaning that a future without the antimicrobials we depend on for protection against infectious disease is imminent. Since peak production and discovery of antibiotics during the golden era of the 1940-70s, few new classes of antibiotics have been identified and introduced to the clinic; of those that have, resistance has rapidly followed.

Nanotechnology offers a means by which new antimicrobials can be developed, or the lifespan of current antimicrobials can be extended. Nanoparticles are loosely defined as particles with at least one dimension smaller than $100 \mathrm{~nm}$; their specific surface area, chemical and biological activity can be tuned for a desired application. As such they have become attractive within a variety of fields including medicine and, in particular, antimicrobial therapy. Nanoparticles with specific surface chemistry and size can intimately interact with the microbial surface mediating an antimicrobial effect that does not necessarily rely on the release of chemically active components. Moreover, nanoparticles can be incorporated into polymers or applied as coatings on surfaces, such as indwelling medical devices, making them extremely versatile; combined with a slow rate of release this means that they offer sustained antimicrobial activity.We have recently demonstrated that chlorhexidine nanoparticles can be deposited on glass, titanium, alginatebased wound dressings and ethylene vinyl acetate and incorporated into dental filling cement (Barbour et al., 2013; Hook et al., 2014; Wood et al., 2014). The presence of the chlorhexidine particles impairs bacterial binding to coated materials as well as mediating bacterial death.
Furthermore, these nanoparticles had sustained efficacy against some of the most notorious healthcare and wound associated pathogens including Pseudomonas aeruginosa and methicillin resistant Staphylococcus aureus.

The development of antimicrobial nanoparticles has primarily focused on metals, which are known to mediate widespread microbial destruction, often facilitated by oxidative damage. Zinc and silver have received the most attention, silver having been incorporated into numerous substrates including wound dressings, clothing and paints in various particulate forms. Metals are well known for their antimicrobial activity, but those that are developed as nanoparticles tend to have greater efficacy and a lower association with the development of resistance, when compared to their chemical counterparts (Azam et al., 2012). Consequently, nanoparticle based antimicrobials offer a versatile and attractive option as a means to deliver effective elimination of pathogens whilst minimising the likelihood of resistance - a welcome addition to our failing arsenal of antimicrobial treatments.

There are, however, some reservations regarding this technology, specifically associated with the toxicity of some metals for eukaryotic cells, such as epithelial and endothelial cells as well as fibroblasts and macrophage (El-Ansary and Al-Daihan, 2009). There remains insufficient data describing the outcome of prolonged human exposure to nanoparticles, as well as potential environmental impacts. Evidence exists demonstrating that some metal nanoparticles impair the biological function of human tissues, organs, cells and proteins. The small size that makes nanoparticles so successful against microorganisms also mediates easy access to the lungs, mucosa, brain and through the skin, where

Corresponding Author: Sarah E. Maddocks, Department of Biomedical Science, Cardiff School of Health Sciences, Cardiff Metropolitan University, UK 
oxidative damage can occur. Oxidative stress can lead to cellular death, membrane and DNA degradation and little is understood about the cumulative impact this could have. Currently one of the major problems is the lack of models to study these effects beyond the study of in vitro cell lines to assess toxicity, with only preliminary in vivo studies that have so far used zebra fish as a model (Asharani et al., 2008). Certainly fuller assessment using more robust models is required prior to large scale application of antimicrobial nanoparticles.

However, we should not overlook the fact that some of our most widely used antibiotics are associated with considerable levels of toxicity. Chloramphenicol, introduced in 1949 was once a first-line agent, but is known to cause myelotoxicity (Yunis, 1989). Consequently it is no longer a first line treatment and is now used primarily in countries where more expensive, less toxic antimicrobials are not an option. For many years we have relied upon a trade-off between antimicrobial efficacy and toxicity to humans, with doses carefully calculated and administered accordingly. As more multidrug resistant bacteria emerge, the medical profession once again has begun employ such antibiotics. In light of the ever decreasing options available to treat infection, newly formulated antimicrobial compounds, including nanoparticles, should perhaps not be overlooked on the basis of potential toxicity.

Until a few months ago, no new classes of antibiotics had been discovered for approximately 20 years and despite this, the frequency at which antimicrobial resistance develops continues to outstrip the rate at which new antibiotics are available (O'Daniel et al., 2014). Consequently novel ways of adapting the antimicrobials that are currently available to us provides an alternative route to ensuring that we remain one step ahead of antimicrobial resistance. The nature of the production of nanoparticle antimicrobials means that they can be manufactured quickly and cheaply. Chemical design and synthesis of new drugs is extremely costly, a factor that is passed onto the consumer meaning that often newer antimicrobial treatments remain the luxury of those nations who can afford them. Antimicrobial resistance is now recognised as a global threat that needs to be met with a global strategy; in essence the world needs to recognise the problem and co-ordinate a means to resolve it. Therefore nanoparticle antimicrobials could go some of the way to enabling better accessibility of treatment in terms of limitations posed by cost.

Whilst no single antimicrobial treatment or strategy will ever resolve the problem of on-going antimicrobial resistance, the development of new antimicrobials, including nanoparticles, will serve to keep us at the fore. Therefore we believe that there is undeniably a place for nanoparticle antimicrobials in the global therapeutic repertoire and as a part of the strategy to ensure that efficacious treatments exist well into the future.

\section{REFERENCES}

Asharani, P.V., W.Y. Lian, Z. Gong and S. Valiyaveettil, 2008. Toxicity of silver nanoparticles in zebrafish models. Nanotechnology, 19: 255102-255102. DOI: 10.1088/0957-4484/19/25/255102

Azam, A., S.A. Ahmed, M. Oves, M.S. Khan and S.S. Habib et al., 2012. Antimicrobial activity of metal oxide nanoparticles against Gram-positive and Gram-negative bacteria: A comparative study. Int. J. Nanimed., 12: 6003-6009. DOI: 10.2147/IJN.S35347

Barbour, M.E., S.E. Maddocks, N.J. Wood and A.M. Collins, 2013. Synthesis, characterization and efficacy of antimicrobial chlorhexidine hexametaphosphate nanoparticles for applications in biomedical materials and consumer products. Int. J. Nanomed., 8: 3507-3519. DOI: 10.2147/IJN.S50140

El-Ansary, A. and S. Al-Daihan, 2009. On the toxicity of therapeutically used nanoparticles: An overview. J. Toxicol., 2009: 754810-754818. DOI: $10.1155 / 2009 / 754810$

Hook, E.R., O.J. Owen, C.A. Bellis, J.A. Holder and D.J. O'Sullivan et al., 2014. Development of a novel antimicrobial-releasing glass ionomer cement functionalized with chlorhexidine hexametaphosphate nanoparticles. J. Nanobiotechnol., 12: 3-3. DOI: 10.1186/1477-315512-3

O’Daniel, P.I., Z. Peng, H. Pi, S.A. Testero and D. Ding et al., 2014. Discovery of a new class of non$\beta$-lactam inhibitors of penicillin-binding proteins with gram-positive antibacterial activity. J. Am. Chem. Soc., 136: 3664-3672. DOI: 10.1021/ja500053x

Wood, R.E., A. Arrizabalaga, M. Camps, S. Fallon and M.J. Iriarte-Chiapussoc et al., 2014. The chronology of the earliest Upper Palaeolithic in northern Iberia: New insights from L'Arbreda, Labeko Koba and La Viña. J. Human Evolut., 69: 91-109. DOI: 10.1016/j.jhevol.2013.12.017

Yunis, A.A., 1989. Chloramphenicol toxicity: 25 years of research. Am. J. Med., 87: 44N-48N. PMID: 2486534 